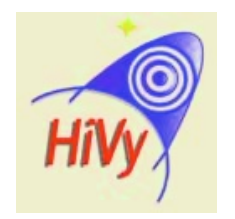

\title{
The HiVy Tool Set
}

\author{
Paula J. Pingree ${ }^{1}$ and Erich Mikk ${ }^{2}$ \\ ${ }^{1}$ Jet Propulsion Laboratory, Pasadena, CA 91109 \\ Paula.J.Pingree@jpl.nasa.gov \\ ${ }^{2}$ Erlangen, Germany \\ Erich.Mikk@epost.de
}

\begin{abstract}
Our aim is to validate mission-specific components of spacecraft flight software designs that are specified using state-charts and translated automatically to the final flight code for the mission. We established an automatic translation tool set from state-charts to the input language of SPIN for the validation of such mission-specific components. To guarantee compliance with autogenerated flight code, our translation tool set preserves the StateFlow ${ }^{\circledR}$ semantics. We are now able to specify and validate portions of mission-critical software design and implementation using the exhaustive exploration techniques of model checking.
\end{abstract}

Keywords: state-charts, model checking, translation, Stateflow, SPIN

\section{Introduction}

The HiVy Tool Set enables model checking for state-charts [1]. This is achieved by translating state-chart specifications into Promela, the input language of the SPIN model checker [2]. The HiVy Tool Set transforms output of the commercial tool Stateflow ${ }^{\circledR}$ provided by The Mathworks. HiVy can also be used independently from Stateflow. An abstract syntax of hierarchical sequential automata (HSA) is provided as an intermediate format for the tool set [3]. The HiVy Tool Set programs include SfParse, sf $2 h s a, h s a 2 p r$ and the HSA merge facility.

\section{Rationale}

The authors of Stateflow adopted the graphical notation of state-charts as proposed by D. Harel [4] but designed a different semantics to this notation. The Statemate by ILogix tool supports the original semantics developed by D. Harel and there are some advances that extend Statemate specified designs to model checking facilities [5], [6]. The following partial list illustrates differences in semantic design between Stateflow and Statemate state-charts, which make clear that Statemate-based tools cannot be used for Stateflow state-chart verification. 
- In Stateflow semantics there is at most one event active at a time. In Statemate semantics any finite number of events are allowed.

- Emitting an event in Stateflow semantics means to pass control to the receiver chart of the event at the moment of emitting the event. In Statemate semantics events are collected until the end of the step and then broadcast to the chart.

- In Stateflow the execution order within an AND-state is determined by the graphical placement of the AND-composed charts. In Statemate all AND-states are executed simultaneously.

- In Stateflow semantics the effect of changing variables takes place immediately. In Statemate semantics a variable change takes effect only at the end of the step.

We propose a new format called hierarchical sequential automata (HSA) that accurately reflects the semantics of Stateflow for implementation within the HiVy Tool Set. Each state-chart is associated with an equivalent hierarchical sequential automaton that consists of a finite set of cooperating sequential automata that can be implemented as parallel processes in Promela. State-chart states, events and variables are encoded as Promela variables and Promela processes change the values of these variables in order to simulate state changes, event generation and variable changes according to the semantics of Stateflow. The observable behavior is defined with respect to the variables representing state-chart states, events and variables. These ideas are very close to those for translating Statemate state-charts to Promela as presented in [6].

\section{Overview of the Tool Set}

\section{Constructing a State-Chart}

A state-chart model of the system to be verified must first be constructed. Access to and general familiarity with the Stateflow application is needed.

Syntactic Restrictions. In order to use the HiVy Tool Set the state-chart must be designed in a sub-set of the Stateflow language. This sub-set does not include:

- Implicit event generation* (See Figures 1 \& 2)

- Inner transitions with the same source and destination*

- Transition actions on transition segments that end on a junction

- History junctions

* Alternate implementations are supported within $\mathrm{HiVy}$

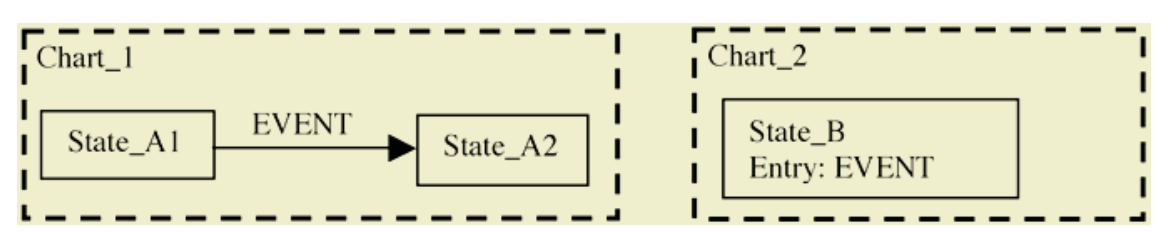

The diagram above represents the following state-chart activity. In Chart_1, State_A1 is active. In Chart_2, State_B is or becomes active. Upon entry into State_B an EVENT is internally generated. This EVENT is sensed by Chart_1 and causes the transition from State_A1 to State_A2 to be made.

Fig. 1. Implicit Event Notation Example 


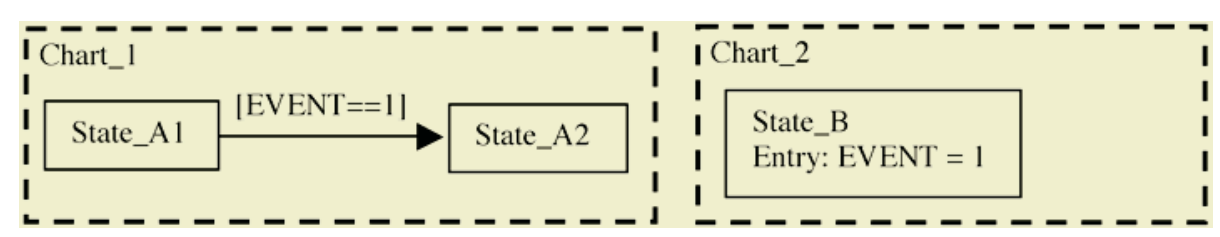

The diagram above represents an equivalent execution to that shown in Figure 1. We set a variable EVENT $=1$ in State_B and create a conditional guard on the transition from State_A1 to State_A2 that evaluates to TRUE when EVENT==1. This notation is suitable for HiVy translation.

Fig. 2. Equivalent Implicit Event Notation

It is significant to note that HiVy supports state-chart events generated by an external environment (e.g., emitted non-deterministically from an included Promela if-loop).

There are additional, simple rules for state-chart model development to support successful translation with HiVy that pertain to scoping (dictation of where nongraphical object types can exist in the hierarchy), support for embedded state-charts or sub-charts, and closed-system verification with SPIN.

\section{Performing Model Translation}

This section describes how to parse Stateflow models to produce the HSA intermediate format, merge sub-charted state-charts with their parent charts (if desired), and generate Promela input for the SPIN model checker.

Parsing \& HSA. Two programs of the HiVy Tool Set: SfParse and sf $2 h s a$ are used to prepare the model file for translation. If parsing is successful, a file is produced that contains an ASCII representation of the abstract syntax tree in HSA-format. HSA is given by a set of events, a set of states, a root state, a set of Boolean variables bvars, a set of integer variables ivars, a set of states denoting the initial configuration iconf, a hierarchy function $h i$, a typing function $t y$, a transition map trmap and OR-state mappings to their initial states initmap [3].

Merging State-Charts. Models consisting of several files may be merged into one HSA file before translating into Promela for SPIN using the HiVy program hsacomplete.

File Generation for SPIN Model Checking. The following files are generated by the HiVy translation program hsa2pr:

- stmodel.pr: the Promela model of the original state-chart.

- prop_list and propositions: the name list and definitions of propositions. One proposition is generated for each state and event. Proposition names are suitable in generating linear temporal logic (LTL) properties for verification.

\section{HiVy Metrics and Optimization}

A Plain Ol' Telephone System (POTS) model was developed to prototype HiVy's translation capability and performance. The Stateflow model contains 36 states and 7 events. The Promela file containing 1300 LOC (including comments) is immediately 
produced by executing the HiVy Tool Set programs. The translated model contains 13 Promela processes. The number of Promela processes directly affects SPIN verification performance. To minimize system compute time and memory utilization, it is desirable to limit the number of processes in a SPIN model. A HiVy optimization algorithm that produces a valid translation to a model with a single Promela process is currently in development. Preliminary model verification metrics as reported by SPIN for the original and optimized Promela POTS models are provided in Table 1 below.

Table 1. POTS Example Metrics

\begin{tabular}{|l|c|c|c|}
\hline Parameter & Original HiVy & HiVy Optimization & Reduction \\
\hline Real/user time & 3.5 seconds & 1.5 seconds & $57 \%$ \\
\hline Total actual memory use & $8.87 \mathrm{Mb}$ & $4.67 \mathrm{Mb}$ & $47 \%$ \\
\hline
\end{tabular}

\section{In Conclusion}

The full capability of the SPIN model checker may be used to verify models generated by HiVy because they yield valid Promela code. The development of our approach for verification of NASA Spacecraft Fault Protection designs has been presented previously [7], [8]. The validity of HiVy generated models for SPIN model checking has been prototyped. State-space explosion effects are the significant challenge to the translation technique. Optimization approaches are under evaluation with initial results showing significant $(>40 \%)$ reduction in verification parameter values.

\section{Acknowledgements}

The authors acknowledge the work of Gordon C. Cucullu III in developing the POTS model. The research described in this paper was carried out at the Jet Propulsion Laboratory, California Institute of Technology, under a contract with the National Aeronautics and Space Administration.

\section{References}

1. The Mathworks Stateflow Users Guide, http://www.mathworks.com

2. G.J. Holzmann. The Model Checker Spin. IEEE Trans. on Software Engineering, 23(5):279295, May 1997. Special issue on Formal Methods in Software Practice.

3. E. Mikk. HSA-Format, private communication 2002.

4. D. Harel, Statecharts: A Visual Formalism for Complex Systems. Science of Computer Programming, ASSP-34(2):362, 1986.

5. E. Mikk, Semantics and Verification of Statecharts. PhD Thesis. Technical Report of Christian-Albrechts-University in Kiel, October 2000

6. E. Mikk, Y. Lakhnech, M. Siegel and G. Holzmann, Implementing Statecharts in PROMELA/SPIN. In Proceedings of the $2^{\text {nd }}$ IEEE Workshop on Industrial-Strength Formal Specification Techniques. pages 90-101, 1999.

7. K. Barltrop, P. Pingree, Model Checking Investigations for Fault Protection System Validation. 2003 International Conference on Space Mission Challenges for Information Technology, June 2003

8. P. Pingree, E. Mikk, G. Holzmann, M. Smith, D. Dams, Validation of Mission Critical Software Design And Implementation Using Model Checking. The 21st Digital Avionics Systems Conference, October 2002. 